PROCEEDINGS OF THE

AMERICAN MATHEMATICAL SOCIETY

Volume 124, Number 12, December 1996, Pages 3677-3687

S 0002-9939(96)03355-2

\title{
EXTINCTION IN NONAUTONOMOUS COMPETITIVE LOTKA-VOLTERRA SYSTEMS
}

\author{
FRANCISCO MONTES DE OCA AND MARY LOU ZEEMAN
}

(Communicated by Linda Keen)

\begin{abstract}
It is well known that for the two species autonomous competitive Lotka-Volterra model with no fixed point in the open positive quadrant, one of the species is driven to extinction, whilst the other population stabilises at its own carrying capacity. In this paper we prove a generalisation of this result to nonautonomous systems of arbitrary finite dimension. That is, for the $n$ species nonautonomous competitive Lotka-Volterra model, we exhibit simple algebraic criteria on the parameters which guarantee that all but one of the species is driven to extinction. The restriction of the system to the remaining axis is a nonautonomous logistic equation, which has a unique solution $u(t)$ that is strictly positive and bounded for all time; see Coleman (Math. Biosci. 45 (1979), 159-173) and Ahmad (Proc. Amer. Math. Soc. 117 (1993), 199205 ). We prove in addition that all solutions of the $n$-dimensional system with strictly positive initial conditions are asymptotic to $u(t)$.
\end{abstract}

\section{INTRODUCTION}

This paper continues Zeeman [15] in an attempt to generalise classical results about Lotka-Volterra systems in the direction suggested by Ahmad [3], Gopalsamy $[7]$ and others $[5,13]$.

Consider a community of $n$ mutually competing species modeled by the nonautonomous Lotka-Volterra system

$$
\dot{x}_{i}(t)=x_{i}(t)\left(b_{i}(t)-\sum_{j=1}^{n} a_{i j}(t) x_{j}(t)\right), \quad i=1, \ldots, n,
$$

where $x_{i}(t)$ is the population size of the $i$ th species at time $t$, and $\dot{x_{i}}$ denotes $\frac{d x_{i}}{d t}$.

Each $k$-dimensional coordinate subspace of $\mathbf{R}^{n}$ is invariant under system (1.1), $(k \in\{1, \ldots, n\})$, and we adopt the tradition of restricting attention to the closed positive cone $\mathbf{R}_{+}^{n}$. We denote the open positive cone by int $\mathbf{R}_{+}^{n}$, and call a vector $x$ positive if $x \in \mathbf{R}_{+}^{n}$, strictly positive if $x \in \operatorname{int} \mathbf{R}_{+}^{n}$. Given $x, y \in \mathbf{R}^{n}$, we write $x \geq y$ to denote that $(x-y)$ is positive.

Received by the editors March 21, 1995.

1991 Mathematics Subject Classification. Primary 34C35, 92D25; Secondary 34A26.

Key words and phrases. Lotka-Volterra, nonautonomous, Liapunov, competition, extinction.

The first author was supported in part by the Division of Mathematics and Statistics at the University of Texas at San Antonio.

The second author was supported in part by the Office of Research Development at the University of Texas at San Antonio.

(c)1996 American Mathematical Society 
The mutual competition between the species dictates that $a_{i j}(t)>0$ for all $i \neq j$, and for all $t$. In addition we assume throughout that for all $i$ and $j, a_{i j}(t)$ and $b_{i}(t)$ are continuous functions, bounded above and below by strictly positive reals. Thus, when we consider system (1.1) restricted to the $i$ th coordinate axis, we have the nonautonomous logistic equation

$$
\dot{x}_{i}(t)=x_{i}(t)\left(b_{i}(t)-a_{i i}(t) x_{i}(t)\right) .
$$

It is well known that an autonomous logistic equation

$$
\dot{x}=x(b-a x)
$$

with $a, b>0$ has a global attractor on int $\mathbf{R}_{+}$at the carrying capacity $x=\frac{b}{a}$. The combined results of Ahmad [3] and Coleman [6], stated as Lemmas 1.1 and 1.2 below, show that in the nonautonomous equation (1.2) the role of the globally attracting carrying capacity of the autonomous equation is played by a well defined canonical solution $x_{i}^{*}(t)$ to which all other solutions converge.

Lemma 1.1 (Ahmad, Coleman). Equation (1.2) has a unique solution $x_{i}^{*}(t)$ which is bounded above and below by strictly positive reals for all $t$.

We call $x_{i}^{*}$ the canonical solution of equation (1.2).

Lemma 1.2 (Coleman). If $u(t), v(t)$ are solutions of (1.2), then $(u(t)-v(t)) \rightarrow 0$ as $t \rightarrow \infty$.

Thus $u(t), v(t) \rightarrow x_{i}^{*}(t)$ as $t \rightarrow \infty$.

It is a classical result that for a two species autonomous competitive LotkaVolterra model with no fixed point in the open positive cone int $\mathbf{R}_{+}^{2}$, one of the species is driven to extinction, whilst the other population stabilises at its own carrying capacity.

In [3], Ahmad proves an analogous result for nonautonomous two-dimensional competitive Lotka-Volterra systems. That is, under the assumption that each of the coefficient functions is continuous and bounded above and below by strictly positive numbers, he gives simple algebraic criteria under which there is no coexistence of the two species. One of the species is driven to extinction, whilst the other species stabilises at the canonical solution of the logistic equation on that axis.

Ahmad and Lazer [4], and Zeeman [15] generalise the classical result in a different direction: to autonomous competitive Lotka-Volterra systems of arbitrary finite dimension $n$. Ahmad and Lazer find algebraic criteria under which one of the $n$ species is driven to extinction, whilst the remaining $(n-1)$ species coexist stably. Zeeman finds algebraic criteria under which $(n-1)$ of the species are driven to extinction, whilst the remaining species stabilises at its own carrying capacity.

In this paper we improve and generalise the results of [15] to the case of nonautonomous competitive Lotka-Volterra systems of arbitrary finite dimension $n$. See also $[5,11]$ for further generalisations of this work which bridge the gap between the results of Ahmad and Lazer, and those of these authors.

In section 2 we state our main result (Theorem 2.1) and compare it with the main result in [15]. In section 3 we give a geometric interpretation of our algebraic hypotheses, and use this to give a geometric sketch of the proof. We make the proof rigorous in sections $4-6$. 


\section{Statement of Result}

Recall that we assume throughout that for all $i$ and $j, a_{i j}(t)$ and $b_{i}(t)$ are continuous functions, bounded above and below by strictly positive reals. To fix notation, let

$$
a_{i j}^{l}=\inf _{t} a_{i j}(t), \quad a_{i j}^{u}=\sup _{t} a_{i j}(t), \quad b_{i}^{l}=\inf _{t} b_{i}(t), \quad \text { and } b_{i}^{u}=\sup _{t} b_{i}(t) .
$$

Note that in the autonomous case, $a_{i j}^{l}=a_{i j}^{u}=a_{i j}(t)$ for all $t$. In section 3 we give a geometric interpretation of inequalities (2.1) of the following theorem, which should help to unravel the subscripts.

Theorem 2.1. Given system (1.1), suppose that

$$
\forall k>1, \quad \exists i_{k}<k \quad \ni \quad \forall j \leq k, \quad \frac{b_{k}^{u}}{a_{k j}^{l}}<\frac{b_{i_{k}}^{l}}{a_{i_{k} j}^{u}} .
$$

Then every trajectory with initial condition in $\operatorname{int} \mathbf{R}_{+}^{n}$ is asymptotic to $x_{1}^{*}$.

In other words, for all strictly positive initial conditions, species $x_{2}, \ldots, x_{n}$ are driven to extinction, whilst species $x_{1}$ stabilises at the unique bounded solution $x_{1}^{*}$ of the logistic equation on the $x_{1}$-axis.

We prove Theorem 2.1 in sections 4,5 and 6 , proving the extinction of species $x_{2}, \ldots, x_{n}$ in section 5 (Theorem 5.1), and the convergence of trajectories to $x_{1}^{*}$ in section 6 (Theorem 6.1).

Allowing for relabeling of the axes, we have:

Corollary 2.2. If there is a permutation $\phi$ of the indices $\{1, \ldots, n\}$ after which system (1.1) satisfies inequalities (2.1), then every trajectory with initial condition in int $\mathbf{R}_{+}^{n}$ is asymptotic to $x_{\phi^{-1}(1)}^{*}$ under the original system.

The following corollaries relate Theorem 2.1 to the results in Ahmad [3] and Zeeman [15].

Corollary 2.3. Given system (1.1), suppose that

$$
\forall k>1, \quad \forall j \leq k, \quad \frac{b_{k}^{u}}{a_{k j}^{l}}<\frac{b_{1}^{l}}{a_{1 j}^{u}} .
$$

Then every trajectory with initial condition in $\operatorname{int}_{+}^{n}$ is asymptotic to $x_{1}^{*}$.

Proof. Corollary 2.3 follows directly from Theorem 2.1 by setting $i_{k}=1$ for each $k$.

Corollary 2.4. Given system (1.1), suppose that

$$
\frac{b_{j}^{u}}{a_{j j}^{l}}<\frac{b_{1}^{l}}{a_{1 j}^{u}} \forall j>1, \quad \text { and } \quad \frac{b_{j}^{l}}{a_{j j}^{u}}>\frac{b_{k}^{u}}{a_{k j}^{l}} \forall k>j .
$$

Then every trajectory with initial condition in $\operatorname{int}_{+}^{n}$ is asymptotic to $x_{1}^{*}$.

Proof.

$$
\frac{b_{k}^{u}}{a_{k j}^{l}}<\frac{b_{j}^{l}}{a_{j j}^{u}} \leq \frac{b_{j}^{u}}{a_{j j}^{l}}<\frac{b_{1}^{l}}{a_{1 j}^{u}} \quad \forall k>j,
$$


and when $j=k$

$$
\frac{b_{k}^{u}}{a_{k j}^{l}}=\frac{b_{j}^{u}}{a_{j j}^{l}}<\frac{b_{1}^{l}}{a_{1 j}^{u}}
$$

Hence Corollary 2.4 follows from Corollary 2.3.

Remark 2.5. It is clear from this proof that the hypotheses of Corollaries 2.4 and 2.6 may be relaxed to permit one of the sets of inequalities to be weak inequalities. In the two-dimensional case, this agrees with the results of Ahmad in [3].

Corollary 2.6 follows directly from Corollary 2.4. We include it to show how Theorem 2.1 improves the main theorem in [15], which is given by applying Corollary 2.6 to the autonomous case.

Corollary 2.6. Given system (1.1), suppose that

$$
\frac{b_{j}^{u}}{a_{j j}^{l}}<\frac{b_{i}^{l}}{a_{i j}^{u}} \forall i<j, \quad \text { and } \quad \frac{b_{j}^{l}}{a_{j j}^{u}}>\frac{b_{i}^{u}}{a_{i j}^{l}} \forall i>j .
$$

Then every trajectory with initial condition in $\operatorname{int}_{+}^{n}$ is asymptotic to $x_{1}^{*}$.

Remark 2.7. It is interesting to compare the relative strengths of the hypotheses of Theorem 2.1 through Corollary 2.6, by considering the application of each to three-dimensional autonomous competitive Lotka-Volterra systems. These systems were studied in [14], and classified into 33 open equivalence classes called nullcline classes. In [15] it was shown that systems in nullcline class 1 are precisely those satisfying the hypotheses of Corollary 2.6, and that this result is far from being sharp, since nullcline classes 2,3,7 and 8 also consist of systems in which all but one species are driven to extinction.

It is straightforward to verify that (permitting permutation of the axes) the hypotheses of Corollary 2.4 are satisfied by systems in nullcline classes 1 and 3 ; the hypotheses of Corollary 2.3 are satisfied by systems in nullcline classes 1, 3 and 7; and the hypotheses of Theorem 2.1 are satisfied by systems in nullcline classes 1 , 2,3 and 7 . Thus we see that although the main result in this paper is considerably stronger than that in [15], even for the autonomous case, it is still not sharp, as it does not apply to the systems in nullcline class 8 .

\section{Geometric interpretation of the inequalities}

The autonomous case. Consider the autonomous competitive Lotka-Volterra system

$$
\dot{x_{i}}=x_{i}\left(b_{i}-\sum_{j=1}^{n} a_{i j} x_{j}\right), \quad i=1, \ldots, n,
$$

satisfying the inequalities

$$
\forall k>1, \quad \exists i_{k}<k \quad \ni \quad \forall j \leq k, \quad \frac{b_{k}}{a_{k j}}<\frac{b_{i_{k}}}{a_{i_{k} j}} .
$$

System (3.1) restricted to the positive $x_{1}$-axis is an autonomous logistic equation with a global attractor at the carrying capacity $\frac{b_{1}}{a_{11}}$. Theorem 2.1 tells us that the 

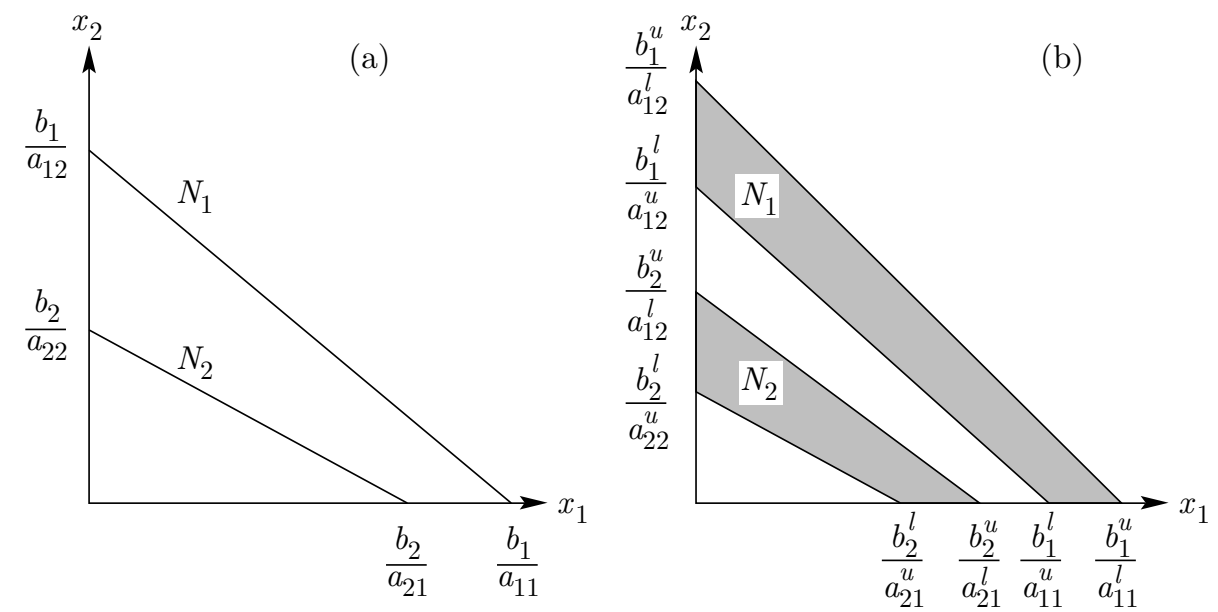

Figure 1. Example of nullclines of (a) an autonomous, and (b) a nonautonomous two-dimensional competitive Lotka-Volterra system satisfying inequalities (2.1).

point $\left(\frac{b_{1}}{a_{11}}, 0, \ldots, 0\right)$ is in fact a global attractor on $\operatorname{int} \mathbf{R}_{+}^{n}$ for the full system (3.1). That is, each of species $x_{2}, \ldots, x_{n}$ is driven to extinction.

We proceed to translate inequalities (3.2) into geometric properties of system (3.1), and to sketch the proof of Theorem 2.1 for the autonomous case, to show how these inequalities inductively lead to the extinction of each $x_{k}$ for $k>1$. A detailed proof of Theorem 2.1 is given in sections 5 and 6 .

The $i$ th nullcline of system (3.1) is the set in $\mathbf{R}_{+}^{n}$ on which $\dot{x}_{i}=0$. It is given by $\left\{x_{i}=0\right\} \cup N_{i}$ where $N_{i}$ is the hyperplane $b_{i}=\sum_{j=1}^{n} a_{i j} x_{j}$, which has positive axial intercepts $\frac{b_{i}}{a_{i j}}$. See Figure 1(a) for a two-dimensional example. Inequalities (3.2) give a partial ordering between the axial intercepts of the hyperplanes $N_{i}$ along each axis, from which we can deduce non-intersection properties of the nullclines in $\mathbf{R}_{+}^{n}$. A geometric analysis of similar non-intersection properties was used to prove the results in [15]. The non-intersection properties corresponding to inequalities (3.2) are considerably weaker than those used in [15], but nevertheless lead to the same conclusion. For example, consider the hyperplanes $N_{n}$ and $N_{i_{n}}$. By inequalities $(3.2)$,

$$
\frac{b_{n}}{a_{n j}}<\frac{b_{i_{n}}}{a_{i_{n} j}}, \quad \forall j \leq n .
$$

Hence $N_{n}$ and $N_{i_{n}}$ are disjoint in $\mathbf{R}_{+}^{n}$. Moreover, $N_{i_{n}}$ lies entirely above $N_{n}$, meaning that $N_{i_{n}}$ is contained in the unbounded component of $\mathbf{R}_{+}^{n} \backslash N_{n}$.

The following lemma is proved in the first half of the proof of Theorem 5.1 (replacing $n$ by $j$ in the proof).

Geometric Lemma 3.1. Given system (3.1), if there exist $i, j$ such that $N_{i}$ lies entirely above $N_{j}$, then species $x_{j}$ is driven to extinction.

Thus $x_{n}$ is driven to extinction by inequalities (3.3). We now restrict attention to the subspace $H^{n-1}$ on which $x_{n}=0$, and we consider the restriction of the 
hyperplanes $N_{(n-1)}$ and $N_{i_{(n-1)}}$ to this subspace. By inequalities (3.2),

$$
\frac{b_{(n-1)}}{a_{(n-1) j}}<\frac{b_{i_{(n-1)}}}{a_{i_{(n-1)} j}} \forall j \leq(n-1),
$$

so $N_{i_{(n-1)}}$ lies entirely above $N_{(n-1)}$ in $H_{+}^{(n-1)}$, and hence $x_{(n-1)}$ is driven to extinction in the subsystem corresponding to $x_{n}=0$.

Similarly, for each $r>1, N_{i_{r}}$ lies entirely above $N_{r}$ in the $r$-dimensional subspace $H^{r}$ on which $x_{(r+1)}, \ldots, x_{n}$ vanish, and hence $x_{r}$ is driven to extinction in the corresponding subsystem.

Thus inequalities (3.2) lead inductively to the extinction of $x_{k}$ for each $k>1$, and hence we prove in Theorem 6.1 that $R_{1}$ is globally attracting on $\operatorname{int} \mathbf{R}_{+}^{n}$.

The nonautonomous case. Now consider the nonautonomous system (1.1) satisfying inequalities (2.1). Define the upper system of system (1.1) to be the autonomous system

$$
\dot{x}_{i}=x_{i}\left(b_{i}^{u}-\sum_{j=1}^{n} a_{i j}^{l} x_{j}\right), \quad i=1, \ldots, n,
$$

and define the lower system by

$$
\dot{x_{i}}=x_{i}\left(b_{i}^{l}-\sum_{j=1}^{n} a_{i j}^{u} x_{j}\right), \quad i=1, \ldots, n .
$$

Then for each $i$, the upper system has $i$ th nullcline $N_{i}^{u}$, as defined above and which meets the $x_{j}$-axis at $\frac{b_{i}^{u}}{a_{i j}^{l}}$. Similarly, the lower system has $i$ th nullcline $N_{i}^{l}$, which meets the $x_{j}$ axis at $\frac{b_{i}^{l}}{a_{i j}^{u}}$.

We use these upper and lower nullclines to define a thickened nullcline $N_{i}$ for system (1.1) by

$$
N_{i}=\left\{x \in \mathbf{R}_{+}^{n} \quad: \quad \exists y \in N_{i}^{l}, z \in N_{i}^{u} \quad \ni \quad y \leq x \leq z\right\}
$$

In other words, $N_{i}$ is the region in $\mathbf{R}_{+}^{n}$ between $N_{i}^{l}$ and $N_{i}^{u}$. See Figure 1(b) for a two-dimensional example.

For each fixed t, the $i$ th nullcline $N_{i}(t)$ of the autonomous system with coefficients $a_{i j}(t), b_{i}(t)$ meets the $x_{j}$-axis at $\frac{b_{i}(t)}{a_{i j}(t)}$. Now,

$$
\frac{b_{i}^{l}}{a_{i j}^{u}}<\frac{b_{i}(t)}{a_{i j}(t)}<\frac{b_{i}^{u}}{a_{i j}^{l}} \quad \forall j=1, \ldots, n,
$$

and hence $N_{i}(t) \subseteq N_{i}$. In other words, the $i$ th nullcline of the autonomous system corresponding to each fixed $t$ is contained in the $i$ th thickened nullcline of the nonautonomous system.

Inequalities (2.1) can now be interpreted, in direct analogy with the autonomous case, as non-intersection properties of the thickened nullclines. For example: $N_{i_{n}}^{l}$ lies entirely above $N_{n}^{u}$, and hence (thickened) $N_{i_{n}}$ lies entirely above (thickened) $N_{n}$. Moreover, the proof of Theorem 5.1 shows that the geometric lemma (Lemma 3.1) holds for the nonautonomous case as well as the autonomous case, and thus the non-intersection properties lead to the extinction of species $x_{2}, \ldots, x_{n}$ just as before. 


\section{A compact attracting REgion}

There are many ways to find a compact attracting region for system (1.1). Lemma 4.1 uses a particularly simple compact attracting region to give coarse bounds on the components of a solution to system (1.1). These bounds are needed for the estimation in sections 5 and 6 . A more delicate and optimal compact attracting region for system (1.1) is found in [16].

Lemma 4.1. If $x(t)$ is a solution of system (1.1) with initial condition in $\operatorname{int}_{+}^{n}$, then there exist $r, \delta>0$ and $T \in \mathbf{R}$ such that for all $t>T$,

$$
\sum_{i=1}^{n} x_{i}(t) \geq \delta \quad \text { and } \quad 0<x_{i}(t) \leq r, \quad \forall i=1, \ldots, n .
$$

Proof. It is clear that the open and closed positive cones are invariant under system (1.1). Now choose

$$
r \geq 2 \max \left\{\frac{b_{i}^{u}}{a_{i j}^{l}}: i, j=1, \ldots, n\right\}, \quad \delta \leq \frac{1}{2} \min \left\{\frac{b_{i}^{l}}{a_{i j}^{u}}: i, j=1, \ldots, n\right\}
$$

and define

$$
S=\left\{x \in \mathbf{R}_{+}^{n}: \delta \leq \sum_{i=1}^{n} x_{i}(t) \leq r\right\} .
$$

We shall show that $S$ is a globally attracting positively invariant compact set for $\mathbf{R}_{+}^{n} \backslash\{0\}$. Then if $x(t)$ is a solution of system (1.1) with initial condition in int $\mathbf{R}_{+}^{n}$, there exists $T \in \mathbf{R}$ such that for all $t>T, x(t) \in S$, and the conclusion follows.

Consider the function $L: \mathbf{R}_{+}^{n} \rightarrow \mathbf{R}_{+}$defined by $L(x)=\sum_{i=1}^{n} x_{i}$. Then $\nabla L=$ $(1, \ldots, 1)$, and the derivative of $L$ along trajectories of system (1.1) is given by

$$
\dot{L}=\nabla L . \dot{x}=\sum_{i=1}^{n} \dot{x}_{i}=\sum_{i=1}^{n} x_{i}(t)\left(b_{i}(t)-\sum_{j=1}^{n} a_{i j}(t) x_{j}(t)\right) .
$$

It is easy to see that $L$ is Liapunov-like outside $S$. That is, by our choice of $\delta$, if $x \in \mathbf{R}_{+}^{n} \backslash\{0\}$ satisfies $\sum_{i=1}^{n} x_{i} \leq \delta$ then $\dot{x}_{i}$ is non-negative for each $i$, and strictly positive for at least one value of $i$. So at each $x$ satisfying $0<L(x) \leq \delta, \dot{L}$ is strictly positive. Similarly, for each $x$ satisfying $L(x) \geq r, \dot{L}$ is strictly negative. Hence $[\delta, r]$ is a compact attracting set for $L$ along each trajectory of system (1.1), and thus $S$ is a compact attracting set for the flow of system (1.1) on $\mathbf{R}_{+}^{n} \backslash\{0\}$.

\section{Extinction of $x_{2}, \ldots, x_{n}$}

Theorem 5.1. If system (1.1) satisfies inequalities (2.1) and $x(t)$ is a solution of system (1.1) with $x\left(t_{0}\right) \in \operatorname{int}_{+}^{n}$ for some $t_{0}$, then for all $i=2, \ldots, n$,

(a) $\quad x_{i}(t) \rightarrow 0$ as $t \rightarrow \infty$, and

(b) $\quad \int_{t_{0}}^{\infty} x_{i}(t) d t<\infty$.

Remark 5.2. Let $H^{k}$ denote the $k$-dimensional subspace on which $x_{k+1}, \ldots, x_{n}$ all vanish. Generalising the method in [15], conclusion (a) of Theorem 5.1 can be proved by inductively applying the Liapunov functions

$$
V_{k}(x)=x_{i_{k}}^{-b_{k}^{u}} x_{k}^{b_{i_{k}}^{l}}
$$


to each $H^{k}$. However, we shall need conclusion (b) to prove that solutions are asymptotic to $x_{1}^{*}$, so we use the idea behind the Liapunov functions $V_{k}$ to develop an integration proof here.

Proof of Theorem 5.1. Let $x(t)$ be a solution of system (1.1) with $x\left(t_{0}\right) \in \operatorname{int} \mathbf{R}_{+}^{n}$ for some $t_{0}$. By Lemma 4.1 we may assume that $x\left(t_{0}\right) \in S$. We prove Theorem 5.1 by induction. First we show that conclusions (a) and (b) hold for $x_{n}(t)$. Let $i=i_{n}$ given by inequalities (2.1). By definition

$$
\frac{\dot{x}_{i}(t)}{x_{i}(t)} \geq b_{i}^{l}-\sum_{j=1}^{n} a_{i j}^{u} x_{j}(t) \quad \text { and } \quad \frac{\dot{x}_{n}(t)}{x_{n}(t)} \leq b_{n}^{u}-\sum_{j=1}^{n} a_{n j}^{l} x_{j}(t)
$$

so

$$
\begin{aligned}
\frac{d}{d t} \ln \left(x_{i}^{-b_{n}^{u}}(t) x_{n}^{b_{i}^{l}}(t)\right) & =b_{i}^{l}\left(\frac{\dot{x}_{n}(t)}{x_{n}(t)}\right)-b_{n}^{u}\left(\frac{\dot{x}_{i}(t)}{x_{i}(t)}\right) \\
& \leq \sum_{j=1}^{n}\left(b_{n}^{u} a_{i j}^{u}-b_{i}^{l} a_{n j}^{l}\right) x_{j}(t) \\
& \leq \max _{j}\left\{b_{n}^{u} a_{i j}^{u}-b_{i}^{l} a_{n j}^{l}\right\} \sum_{j=1}^{n} x_{j}(t) .
\end{aligned}
$$

Hence, for $t>t_{0}$

$$
\frac{d}{d t} \ln \left(x_{i}^{-b_{n}^{u}}(t) x_{n}^{b_{i}^{l}}(t)\right) \leq \max _{j}\left\{b_{n}^{u} a_{i j}^{u}-b_{i}^{l} a_{n j}^{l}\right\} \delta<\delta_{n}
$$

for some real $\delta_{n}<0$, by Lemma 4.1 and inequalities (2.1). Integrating this equation we have

$$
\left.\ln \left(x_{i}^{-b_{n}^{u}}(t) x_{n}^{b_{i}^{l}}(t)\right)\right|_{t_{0}} ^{t}<\delta_{n}\left(t-t_{0}\right)
$$

and so for $t>t_{0}$

$$
x_{n}^{b_{i}^{l}}(t)<C e^{\delta_{n}\left(t-t_{0}\right)}, \quad \text { where } C=\frac{x_{n}^{b_{i}^{l}}\left(t_{0}\right)}{x_{i}^{b_{n}^{u}}\left(t_{0}\right)} r^{b_{n}^{u}} .
$$

Thus

$$
x_{n}(t)<K_{n} e^{\varepsilon_{n}\left(t-t_{0}\right)}, \quad \forall t>t_{0},
$$

where $\varepsilon_{n}=\frac{\delta_{n}}{b_{i}^{l}}<0$ and $K_{n}>0$. Conclusions (a) and (b) for $x_{n}$ follow directly.

We now prove that for $1<r<n, x_{r} \rightarrow 0$ as $t \rightarrow \infty$ under the assumption that for $r<j \leq n, x_{j} \rightarrow 0$ as $t \rightarrow \infty$. The method is essentially the same as that used above for $x_{n}$. Now let $i=i_{r}$ given by inequalities (2.1). Then

$$
\frac{\dot{x}_{i}(t)}{x_{i}(t)} \geq b_{i}^{l}-\sum_{j=1}^{n} a_{i j}^{u} x_{j}(t) \quad \text { and } \quad \frac{\dot{x}_{r}(t)}{x_{r}(t)} \leq b_{r}^{u}-\sum_{j=1}^{n} a_{r j}^{l} x_{j}(t)
$$


SO

$$
\begin{aligned}
\frac{d}{d t} \ln \left(x_{i}^{-b_{r}^{u}}(t) x_{r}^{b_{i}^{l}}(t)\right) & =b_{i}^{l}\left(\frac{\dot{x}_{r}(t)}{x_{r}(t)}\right)-b_{r}^{u}\left(\frac{\dot{x}_{i}(t)}{x_{i}(t)}\right) \\
& \leq \sum_{j=1}^{n}\left(b_{r}^{u} a_{i j}^{u}-b_{i}^{l} a_{r j}^{l}\right) x_{j}(t) \\
& =\sum_{j=1}^{r}\left(b_{r}^{u} a_{i j}^{u}-b_{i}^{l} a_{r j}^{l}\right) x_{j}(t)+\sum_{j=r+1}^{n}\left(b_{r}^{u} a_{i j}^{u}-b_{i}^{l} a_{r j}^{l}\right) x_{j}(t) .
\end{aligned}
$$

By inequalities (2.1) each term in the first summation is strictly negative. The inequalities do not give us control over the sign of the second summation. Instead we use the assumption that $x_{j} \rightarrow 0$ as $t \rightarrow \infty$, for $j>r$, as follows.

Firstly, note that for $t$ sufficiently large $\sum_{j=1}^{r} x_{j}(t)>\frac{\delta}{2}$, where $\delta$ is given by Lemma 4.1. Secondly, choose $\nu>0$ such that $\nu<\left|\max _{j \leq r}\left(b_{r}^{u} a_{i j}^{u}-b_{i}^{l} a_{r j}^{l}\right) \frac{\delta}{2}\right|$, and note that for $t$ sufficiently large $\sum_{j=r+1}^{n}\left(b_{r}^{u} a_{i j}^{u}-b_{i}^{l} a_{r j}^{l}\right) x_{j}(t)<\nu$. Thus there exists $t_{r} \in \mathbf{R}$ such that for $t>t_{r}$

$$
\frac{d}{d t} \ln \left(x_{i}^{-b_{r}^{u}}(t) x_{r}^{b_{i}^{l}}(t)\right)<\max _{j \leq r}\left(b_{r}^{u} a_{i j}^{u}-b_{i}^{l} a_{r j}^{l}\right) \frac{\delta}{2}+\nu=\delta_{r}<0 .
$$

Integrating this equation we have

$$
\left.\ln \left(x_{i}^{-b_{r}^{u}}(t) x_{r}^{b_{i}^{l}}(t)\right)\right|_{t_{r}} ^{t}<\delta_{r}\left(t-t_{r}\right)
$$

and so for $t>t_{r}$

$$
x_{r}(t)<K_{r} e^{\varepsilon_{r}\left(t-t_{r}\right)},
$$

where $\varepsilon_{r}=\frac{\delta_{r}}{b_{i}^{t}}<0$ and $K_{r}>0$. Conclusions (a) and (b) follow directly.

\section{Convergence to $x_{1}^{*}$}

Recall from section 1 that $x_{1}^{*}$ is the canonical solution to the nonautonomous logistic equation obtained by restricting system (1.1) to the $x_{1}$-axis.

Theorem 6.1. If system (1.1) satisfies inequalities (2.1) and $x(t)$ is a solution of system (1.1) with $x\left(t_{0}\right) \in \operatorname{int} \mathbf{R}_{+}^{n}$ for some $t_{0}$, then $x_{1}(t) \rightarrow x_{1}^{*}(t)$ as $t \rightarrow \infty$.

Proof. By Lemma 4.1, we may assume that $x\left(t_{0}\right) \in S \cap \operatorname{int} \mathbf{R}_{+}^{n}$. Then for all $t>t_{0}$, $x(t) \in S$ and $x_{1}(t)$ is bounded above and below by positive constants. Let $u_{1}(t)$ be a solution of the nonautonomous logistic equation (1.2) such that $u_{1}\left(t_{0}\right) \geq x_{1}\left(t_{0}\right)$. Then $u_{1}(t)>x_{1}(t)$ for all $t>t_{0}$ (see Ahmad [1], Lemma 2.8 or Tineo and Alvarez [12], Proposition 2.1), and $u_{1}(t)$ is bounded (Lemmas 1.1 and 1.2). We now follow a technique similar to that used in the proof of the previous theorem to compare $u_{1}(t)$ and $x_{1}(t)$ as $t \rightarrow \infty$ :

$$
\begin{aligned}
\frac{d}{d t}\left(\ln \frac{x_{1}(t)}{u_{1}(t)}\right) & =\frac{\dot{x}_{1}(t)}{x_{1}(t)}-\frac{\dot{u}_{1}(t)}{u_{1}(t)} \\
& =a_{11}(t)\left(u_{1}(t)-x_{1}(t)\right)-\sum_{j=2}^{n} a_{1 j}(t) x_{j}(t)
\end{aligned}
$$


so

$$
u_{1}(t)-x_{1}(t) \leq \frac{1}{a_{11}^{l}}\left(\frac{d}{d t}\left(\ln \frac{x_{1}(t)}{u_{1}(t)}\right)+\sum_{j=2}^{n} a_{1 j}(t) x_{j}(t)\right)
$$

Integrating this inequality we have

$$
\begin{aligned}
\int_{t_{0}}^{t}\left(u_{1}(t)-x_{1}(t)\right) d t & \leq \frac{1}{a_{11}^{l}}\left(\left.\ln \frac{x_{1}(t)}{u_{1}(t)}\right|_{t_{0}} ^{t}+\sum_{j=2}^{n} \int_{t_{0}}^{t} a_{1 j}(t) x_{j}(t) d t\right) \\
& \leq \frac{1}{a_{11}^{l}}\left(\ln \left(\frac{x_{1}(t) u_{1}\left(t_{0}\right)}{u_{1}(t) x_{1}\left(t_{0}\right)}\right)+\sum_{j=2}^{n} a_{1 j}^{u} \int_{t_{0}}^{t} x_{j}(t) d t\right) \\
& <K<\infty,
\end{aligned}
$$

where $K$ is some constant independent of $t$, since $x_{1}(t), u_{1}(t)$ are bounded by positive constants, and for $j>1, \int_{t_{0}}^{\infty} x_{j}(t) d t<\infty$ (Theorem 5.1). Thus

$$
\int_{t_{0}}^{\infty}\left(u_{1}(t)-x_{1}(t)\right) d t<\infty
$$

and so

$$
x_{1}(t) \rightarrow u_{1}(t) \text { as } t \rightarrow \infty,
$$

since $u_{1}(t)-x_{1}(t)$ is a non-negative differentiable function such that $\dot{u}_{1}(t)-\dot{x}_{1}(t)$ is bounded on $\left[t_{0}, \infty\right)$. Moreover, by Lemma 1.2

$$
u_{1}(t) \rightarrow x_{1}^{*}(t) \text { as } t \rightarrow \infty
$$

and hence

$$
x_{1}(t) \rightarrow x_{1}^{*}(t) \quad \text { as } t \rightarrow \infty
$$

Theorem 2.1 is now a corollary of Theorems 5.1 and 6.1 .

\section{ACKNOWLEDGMENT}

We would like to thank Shair Ahmad for introducing us to this problem and for helpful discussions.

\section{REFERENCES}

[1] S. Ahmad. Convergence and Ultimate Bounds of Solutions of the Nonautonomous VolterraLotka Competition Equations, J. Math. Anal. Appl. 127 (1987), 377-387. MR 89a:92032

[2] S. Ahmad. On Almost Periodic Solutions of the Competing Species Problems, Proc. Amer. Math. Soc. 102 (1988), 855-861. MR 89f:92055

[3] S. Ahmad. On the Nonautonomous Volterra-Lotka Competition Equations, Proc. Amer. Math. Soc. 117 (1993), 199-205. MR 93c:34109

[4] S. Ahmad and A. C. Lazer. One Species Extinction in an Autonomous Competition Model, Proc. First World Congress Nonlinear Analysis, Walter DeGruyter, Berlin, 1995.

[5] S. Ahmad and A. C. Lazer On the Nonautonomous N-Competing Species Problem, Appl. Anal. (1995) To appear.

[6] B. D. Coleman. Nonautonomous Logistic Equations as Models of the Adjustment of Populations to Environmental Change, Math. Biosci. 45 (1979), 159-173. MR 80f:92012

[7] K. Gopalsamy. Globally Asymptotic Stability in a Periodic Lotka-Volterra System, J. Math. Anal. Appl. 159 (1985), 44-50. MR 86f:34094 
[8] M. W. Hirsch. Systems of Differential Equations that are Competitive or Cooperative. III: Competing Species, Nonlinearity 1 (1988), 51-71. MR 90d:58070

[9] J. Hofbauer and K. Sigmund. The Theory of Evolution and Dynamical Systems. Cambridge Univ. Press, Cambridge, 1988. MR 91h:92019

[10] R. M. May. Stability and Complexity in Model Ecosystems. Princeton Univ. Press, Princeton, NJ, 1975.

[11] F. Montes de Oca and M. L. Zeeman. Balancing Survival and Extinction in Nonautonomous Competitive Lotka-Volterra Systems, J. Math. Anal. Appl. 192 (1995), 360-370. MR 96c:92017

[12] A. Tineo and C. Alvarez. A Different Consideration about the Globally Asymptotically Stable Solution of the Periodic n-Competing Species Problem, J. Math. Anal. Appl. 159 (1991), 4450. MR 93d:34080

[13] A. Tineo. On the Asymptotic Behaviour of some Population Models, J. Math. Anal. Appl. 167 (1992), 516-529. MR 93g:92027

[14] M. L. Zeeman. Hopf Bifurcations in Competitive Three-Dimensional Lotka-Volterra Systems, Dynamics Stability Systems 8 (1993), 189-217. MR 94j:34044

[15] M. L. Zeeman. Extinction in Competitive Lotka-Volterra Systems, Proc. Amer. Math. Soc. 123 (1995), 87-96. MR 95c:92019

[16] M. L. Zeeman. Thickened Carrying Simplices in Nonautonomous Competitive Lotka-Volterra Systems, To appear.

Universidad Centroccidental, Lisandro Alvarado, Barquisimeto, Venezuela

Division of Mathematics and Statistics, University of Texas at San Antonio, San Antonio, Texas 78249-0664

E-mail address: zeeman@ringer.cs.utsa.edu 